\title{
Korrekturosteotomien an der Hand
}

\author{
Thomas Pillukat, Karlheinz Kalb, Jörg van Schoonhoven
}

\section{Zusammenfassung}

In Fehlstellung verheilte Frakturen können an der Hand Form und Funktion ernsthaft beeinträchtigen. Verkürzung, Achsabweichung in der frontalen und sagittalen Ebene sowie Rotation sind die Grundelemente aller Fehlstellungen und kommen isoliert oder in Kombination vor. In Fällen mit entsprechender Funktionseinschränkung ist daher eine Korrekturosteotomie erforderlich. Zuklappende Osteotomien benötigen keine Knochentransplantate, stellen jedoch die Länge nicht wieder her. Aufklappende Osteotomien erfordern meistens eine Knochentransplantation. Derotierende Osteotomien sind technisch schwierig im Hinblick auf verbleibende Rotationsfehlstellungen. Geeignete Implantate an der Hand sind Kirschner-Drähte, intraossäre Drähte, zu bevorzugen sind aus Präzisions- und Stabilitätsgründen Miniplatten und Schrauben. Eine frühzeitige Korrektur innerhalb der ersten 10 Wochen ergibt bessere Resultate als spätere Operationen. Achsenknicke an den Mittelhandknochen lassen sich durch auf- oder zuklappende Osteotomien im ehemaligen Frakturbereich beseitigen, Rotationsfehlstellungen durch derotierende Osteotomien. Angulation und Rotationsfehlstellungen an den Phalangen werden mit einer lateralen oder dorsal aufklap- penden Osteotomie, falls erforderlich mit gleichzeitiger Derotation, korrigiert. Intraartikuläre Fehlstellungen lassen sich bei frühzeitigem Eingreifen durch eine Osteotomie im Frakturspalt beheben. Um die Fingerbeweglichkeit zu verbessern, werden begleitende Sehnenadhäsionen und Gelenkkontrakturen häufig mitbehandelt. Osteotomieabhängige Komplikationen sind persistierende Deformitäten, eine verzögerte Knochenbruchheilung, Implantatversagen, persistierende Einsteifung und Schmerzen sowie dystrophe Reaktionen. In Abhängigkeit von Alter, Beruf, Aktivitätsniveau und Schwere der Deformität ist bei ausgewählten Patienten die Korrekturosteotomie an der Hand ein wertvolles Werkzeug, um die Handfunktion wiederherzustellen.

\section{Corrective Osteotomies in the Hand}

In the hand, fracture malunion may severely compromise form and function. Shortening, angulation in the frontal and sagittal planes and rotation are the principal elements of deformities and occur isolated or in combination. If the function of the hand is compromised, corrective osteotomies are required. Closing wedge osteotomies do not require bone grafting but poorly restore length. Opening wedge procedures require bone grafting. Derotation osteotomy is critical with regard to persistent rotational deformity. Suitable implants in the hand are K-wires, intraosseous wires, miniplates and screws. A stable osteosynthesis is preferably performed to allow early mobilisation and to avoid the problems of tendon adhesions or joint contractures associated with postoperative immobilisation. Early osteotomy within ten weeks after the injury achieves more favourable results than later operations. Angular and combined angular and rotational deformities of the metacarpals can be corrected by a closing wedge osteotomy at the site of the malunion. Angular and combined angular and rotational deformities of the proximal phalanx may be treated by dorsal or lateral opening or closing wedge osteotomies with derotation if necessary. Articular malunions may be treated with intraarticular corrective osteotomies to restore joint alignment. To improve finger motion, concomitant tendon adhesions and joint contractures are frequently addressed. Osteotomyrelated complications are persistent deformity, delayed union, implant failure, infection, persistent stiffness, dystrophy and persistent pain. Depending on age, profession, activities of daily life and severity of the deformity, corrective osteotomies in the hand are a valuable tool to restore hand function.

\section{Einleitung}

40\% aller Frakturen an der oberen Extremität betreffen die Mittelhandknochen und die Phalangen. Viele dieser Frakturen sind instabil und erfordern eine ausreichende Reposition und Stabilisation.

OP-JOURNAL 2011; 27: 222-227

(c) Georg Thieme Verlag KG Stuttgart · New York DOI http://dx.doi.org/10.1055/s-0031-1298177
Gelingt dies nicht, können die Frakturen in Fehlstellung ausheilen. In Fehlstellung konsolidierte Frakturen sind zwar klinisch stabil, können aber durch ihre Lokalisation, die Art und das Ausmaß der Fehlstellung die Funktion und Ästhetik der gesamten Hand wesentlich beeinträchtigen. Eine Korrektur durch eine Osteotomie ist indiziert, wenn die Handund Fingerfunktion beeinträchtigt ist oder Schmerzen verursacht werden. Eine Korrektur aus ästhetischen Grün- den allein sollte dagegen nur mit größter Zurückhaltung erfolgen.

Korrekturen sollten frühzeitig, d.h. möglichst vor Ablauf der 10. Woche, durchgeführt werden. In diesem Zeitraum lässt sich die Korrektur in der noch identifizierbaren Frakturlinie vornehmen. Das Kallusgewebe ist noch relativ weich und eine Knochentransplantation ist in vielen Fällen nicht erforderlich. Sehnenverklebungen und Gelenkkontrakturen, 


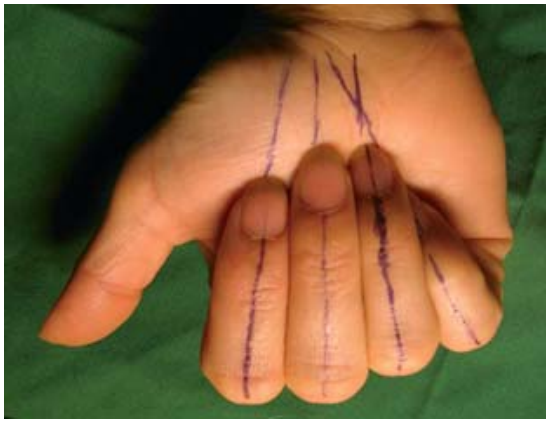

Abb. 1 Alle Strahlen zeigen auf den distalen Kahnbeinpol.

die sich aufgrund der Fehlstellung entwickeln können, werden vermieden oder reduziert. In veralteten Fällen wird die Korrektur nach Ausreifung des Bindegewebes, d.h. frühestens 6 Monate nach dem Unfallereignis, durchgeführt und die Handfunktion in diesem Zeitraum durch eine intensive Übungstherapie optimiert. Wichtig ist, dass eine freie Beweglichkeit der angrenzenden Gelenke besteht bei freiem Durchzug der beteiligten Sehnen. Wird dies nicht vor der Korrekturoperation erreicht, so kann die postoperative Immobilisation zu einer weiteren Verschlechterung des Bewegungsumfangs führen.

Prinzipiell lassen sich folgende Formen der Deformität unterscheiden:

1. Verkürzung

2. Drehfehler (Rotationsfehler)

3. Achsabweichung (Knickbildung)

4. Kombinierte Deformitäten

In der Praxis kann durchaus eine Diskrepanz zwischen dem radiologischen Ausmaß der Fehlstellung und der klinischen Symptomatik bestehen. Hand und Finger verfügen teilweise über eine erstaunliche Adaptationsfähigkeit und können kleinere, manchmal auch größere Fehlstellungen gut kompensieren. Da bei kombinierten Deformitäten meistens eine Fehlstellungsart im Vordergrund steht, ist eine genaue Analyse erforderlich. Die Planung von Korrekturoperationen ist nur anhand einer guten präoperativen Röntgendiagnostik möglich. Voraussetzungen für Korrekturen am Fingerskelett (Phalangen) sind exakt eingestellte Aufnahmen in anteroposteriorer und streng seitlicher Projektion. An den Mittelhandknochen sind Projektionen in 3 Ebenen, d.h. anteroposterior, schräg und streng seitlich, erforderlich. In Zweifelsfällen kann eine Computertomografie weiterhelfen. Intraartikuläre Fehlstellungen sind häufig erst durch die CT-Untersuchung genau zu analysieren. Das Glei-

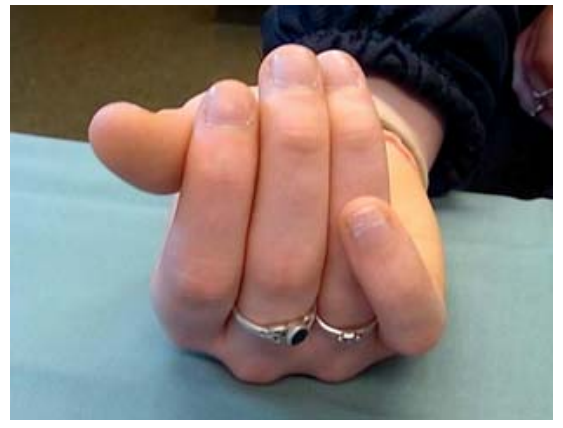

Abb. 2 Überkreuzen des Kleinfingers durch Drehfehler.

che gilt für Fehlstellungen an Mittelhandknochen, wenn die seitliche Projektion wegen Überlagerung mehrerer Mittelhandknochen nicht auswertbar ist.

\section{Fehlstellungen an den Mittelhandknochen}

Klinische Zeichen der Fehlstellung bei Achsabweichungen können ein Verlust der Knöchelkontur, eine diffuse Schwäche und Krampfneigung, ein tastbarer oder sichtbarer Knick des Mittelhandknochens am Handrücken sowie eine Pseudokrallenstellung und ein Streckdefizit in den Grundgelenken sein. In der Hohlhand ist der Kopf des Mittelhandknochens unter Umständen prominent tastbar.

Bei gestreckten Fingern kann ein Rotationsfehler oft nur durch eine unterschiedliche Ausrichtung des Fingernagels festgestellt werden. Beim Faustschluss fehlt die normale Ausrichtung der Fingerachsen auf den distalen Skaphoidpol (Abb. 1). Erst bei Beugung des Fingers wird die Abweichung von der normalen Bewegungsrichtung z.B. auch durch ein Überlappen mit Nachbarfingern sichtbar (Abb. 2).

\section{Verkürzungen}

In Fehlstellung verheilte Spiral- und Schrägfrakturen können zur direkten Verkürzung des Mittelhandknochens führen. Verkürzungen können auch funktionell durch eine Knickbildung entstehen (siehe Achsabweichung). Die Verkürzung des Knochens führt durch die relative Überlänge der Strecksehnen zu Streckdefiziten in den Grundgelenken. Je $2 \mathrm{~mm}$ Verkürzung verursachen ein Streckdefizit von je $7^{\circ}$ und einen Kraftverlust von je $8 \%$. Auf die Funktion der Beugesehnen wirkt sich die Verkürzung dagegen kaum aus.

\section{Achsabweichungen}

Knickbildungen können sowohl in der Frontalebene als auch in der Sagittalebene auftreten. Beide Formen können funktionelle und ästhetische Beeinträchtigungen hervorrufen.

Schaftfrakturen und subkapitale Frakturen der Mittelhandknochen zeigen in der Regel eine Knickbildung in der sagittalen Ebene, deren Scheitelpunkt auf der Streckseite liegt. Nur bei einer Knickbildung im Schaftbereich von weniger als $10^{\circ} \mathrm{im} \mathrm{2.} \mathrm{und} \mathrm{3.} \mathrm{Mittelhandknochen} \mathrm{so-}$ wie $20^{\circ} \mathrm{im} 4$. und $30^{\circ} \mathrm{im} \mathrm{5}$. Mittelhandknochen ist mit einer normalen Funktion zu rechnen. Dieses Ausmaß entspricht der Kompensationsfähigkeit in den Fingergrundgelenken. Die 2. und 3. Mittelhandknochen sind relativ straff mit der Handwurzel verbunden. Diese Strahlen können Fehlstellungen durch Achsabweichungen weniger kompensieren als der 4. und 5. Strahl, die deutlich mobiler mit der Handwurzel verbunden sind. Auch die Lage des Achsknicks ist für die Funktionseinschränkungen bedeutsam. So wird von einigen Autoren angegeben, dass bei subkapitalen Frakturen selbst eine Knickbildung bis $70^{\circ}$ gut kompensiert werden kann. Achsknicke in der Frontalebene sind nur in geringerem Maße und solange tolerabel, wie die Fingerfunktion nicht beeinträchtigt ist. Die randständigen 2. und 5. Strahlen kompensieren diese Fehlstellungen besser als die zentralen 3 . und 4 . Strahlen.

\section{Rotationsfehlstellungen}

Schrägfrakturen und auch Spiralfrakturen können entweder zur isolierten Rotationsfehlstellung führen oder sind mit Verkürzungen kombiniert.

Die Haltebänder zwischen den Köpfen der Mittelhandknochen stabilisieren dabei in gewissem Ausmaß die distalen Fragmente. Dieser Effekt ist stärker bei den zentralen Strahlen als den äußeren Strahlen, weil die Köpfe dieser Mittelhandknochen auf beiden Seiten durch diese Bänder verankert werden.

Während Knickbildungen in geringem Ausmaß durch die angrenzenden Gelenke kompensiert werden können, werden Drehfehler unmittelbar nach distal auf den Finger übertragen und beeinträchtigen die Gesamtfunktion des Fingers. Bereits eine geringe Rotationsfehlstellung kann eine deutliche funktionelle Beein- 


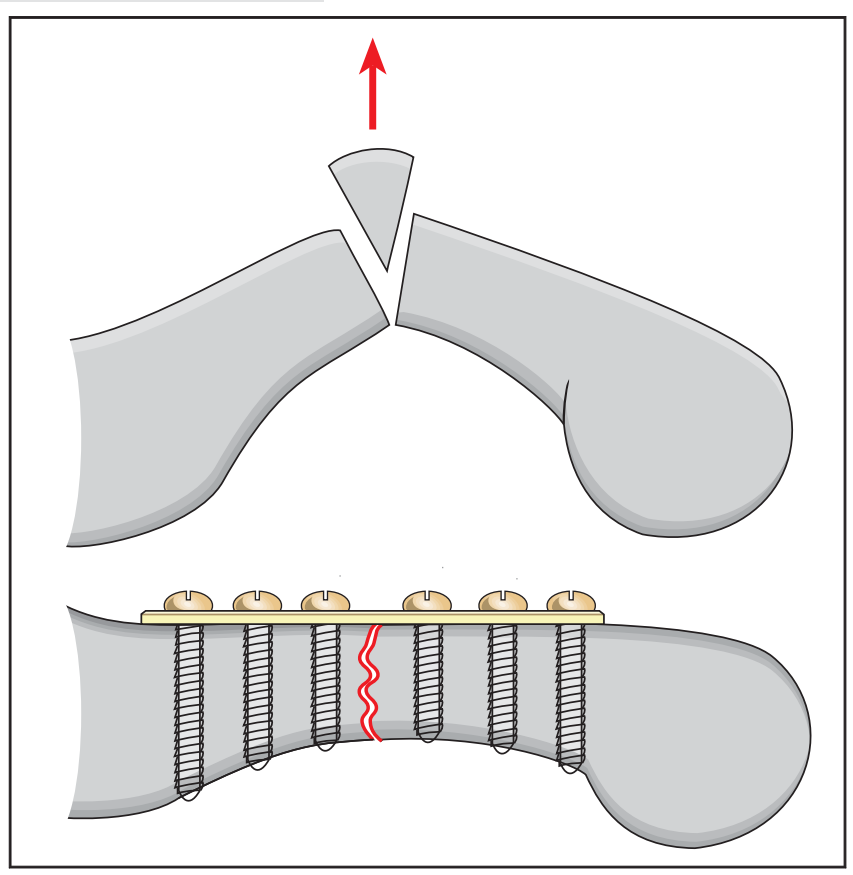

Abb. 3 Zuklappende Osteotomie am Mittelhandknochen. Exzision eines Knochenkeils. Ein Knochentransplantat ist nicht erforderlich.

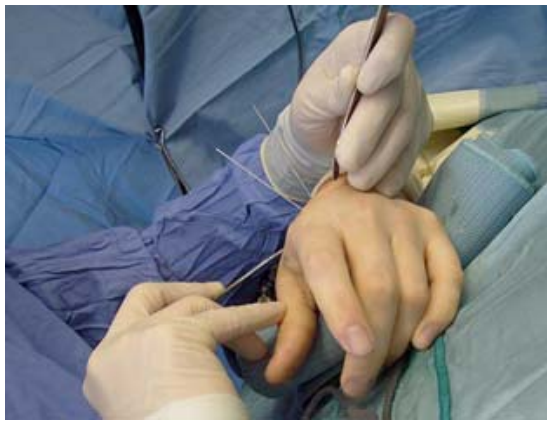

Abb. 4 Präoperativer Rotationsfehler 2. Mittelhandknochen.

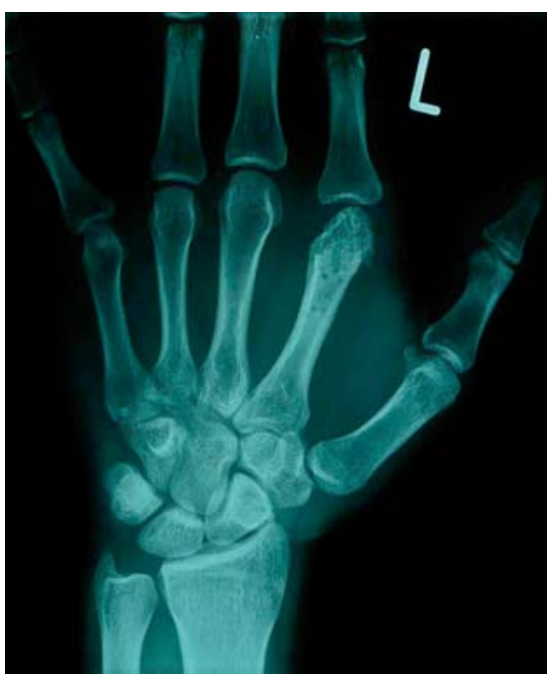

Abb. 5 Fehlverheilter 2. Mittelhandknochen a.-p. präoperativ. gen werden gut toleriert. Die Stabilisation erfolgt durch Miniplatten, die von der Streckseite her angelegt werden.

Die Korrektur eines Drehfehlers (Abb. 49) am Mittelhandknochen kann im ehemaligen Frakturverlauf, aber auch proximal an der Basis des betroffenen Mittelhandknochens erfolgen. Bei einer Kombination von Rotation und Knickbildung sollte die Korrekturosteotomie im Frakturbereich durchgeführt werden, weil eine proximale Osteotomie in diesem Fall die Achsfehlstellung nicht beseitigt. Liegt ausschließlich ein Drehfehler vor, so ist die Korrektur im proximalen Schaftbereich zu bevorzugen. Vorteilhaft ist bei einer Korrektur im metaphysären Bereich die bessere Heilungstendenz, während im diaphysären Abschnitt wegen des kleineren Querschnitts, des höheren Anteils von kortikalem Knochen und der periostalen Denudierung die Knochenbruchheilung ungünstiger verläuft. Als Osteosyntheseverfahren kommen prinzipiell gekreuzte KirschnerDrähte oder Platten und Schrauben infrage. Die Plattenosteosynthese ist jedoch wegen einer wesentlich höheren
Primärstabilität und einer präziseren Fixation der Fragmente eindeutig zu bevorzugen. Diese Präzision ist bei einer Korrekturoperation besonders wichtig. Die höhere Stabilität der Plattenosteosynthese gestattet außerdem einen früheren Beginn der Mobilisation.

\section{Fehlstellungen an den Phalangen}

Die Deformitäten sind häufig multidirektional, d.h. sie stellen eine Kombination verschiedener Formen dar. Beug- und Strecksehnenapparat bilden an den Phalangen ein komplexes System von Sehnenansätzen. Je nach Lage der Sehnenansätze zur Fraktur führt der kontinuierliche Muskelzug bei einfachen Querfrakturen zu charakteristischen Fehlstellungsmustern (Abb. 10). Dieses Prinzip wirkt auch bei komplizierten Frakturen und sollte nicht außer Acht gelassen werden. Bei Ausheilung in Fehlstellung finden sich klinisch Knickbildungen und Rotationsfehler der Finger. 


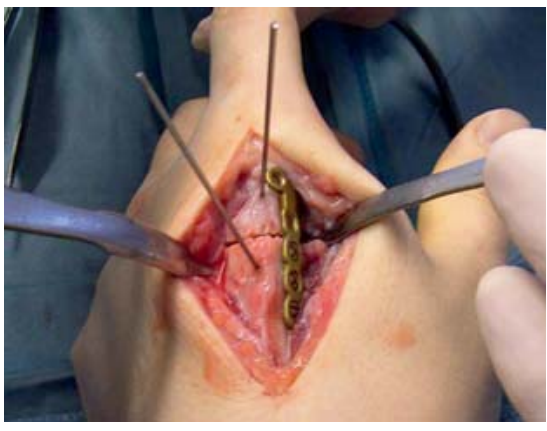

Abb. 6 Derotierende Osteotomie im diaphysären Bereich. Der Rotationsfehler ist noch nicht korrigiert. Die Kirschner-Drähte dienen als Zeiger für die Stellung der Fragmente zueinander.

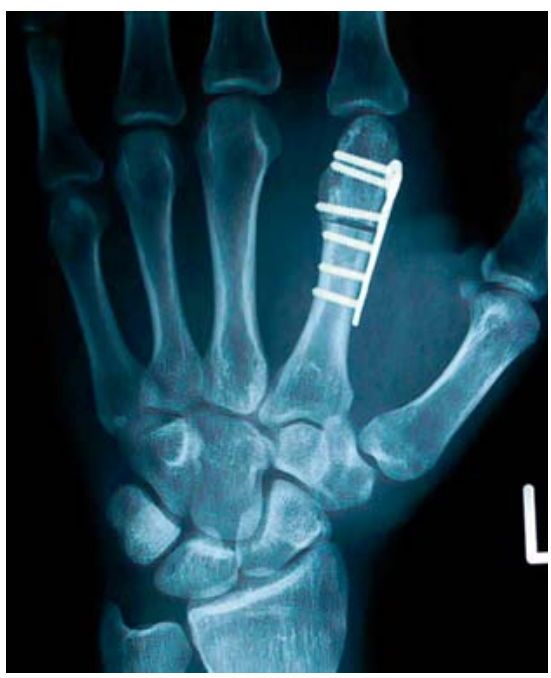

Abb. 9 Korrigierter 2. Mittelhandknochen mit Osteosyntheseplatte.

Die antero-posteriore Röntgenaufnahme vermittelt oft den Eindruck einer scheinbaren anatomischen Position. Die seitliche Aufnahme ist daher unverzichtbar, um eine evtl. verbliebene Knickbildung nach palmar oder dorsal ausreichend beurteilen zu können. Wenn die Angulation mehr als $15^{\circ}$ beträgt, kann sich die Verkürzung funktionell auswirken. In dieser Situation wird nicht mehr die vollständige Streckung im Mittelgelenk erreicht. Das permanente Streckdefizit kann zur Entwicklung einer Pseudokrallenfehlstellung im Grundgelenk führen, wenn das Mittelgelenk, was häufig geschieht, eine fixierte Beugekontraktur entwickelt. Die Beugekontraktur verbleibt auch nach Korrektur der Fehlstellung und macht evtl. weitere Eingriffe erforderlich. Die Nähe der Beugesehne zum Frakturbereich erhöht darüber hinaus das Risiko von Adhäsionen. Gelenkfrakturen können neben Achsabweichungen und Drehfehlern den Verlust der Gelenkkongruenz bedeuten.

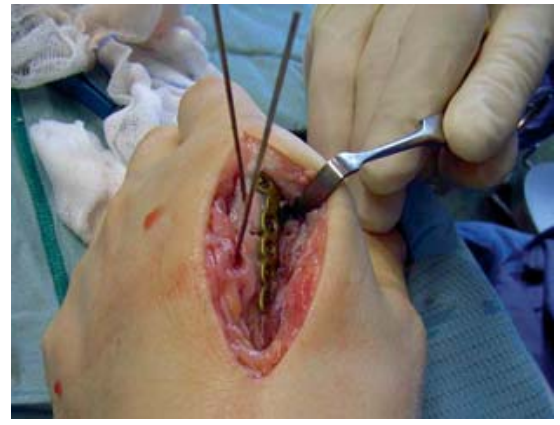

Abb. 7 Korrektur der Rotationsfehlstellung. Beachte den Positionswechsel der KirschnerDrähte.

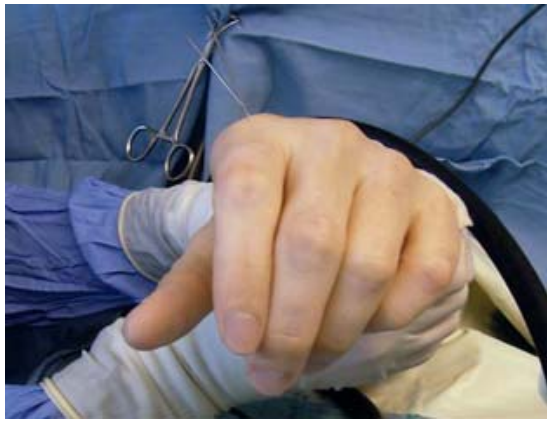

Abb. 8 Klinisches Bild nach der Korrektur. Die Überlappung des Zeige- und Mittelfingers ist aufgehoben.

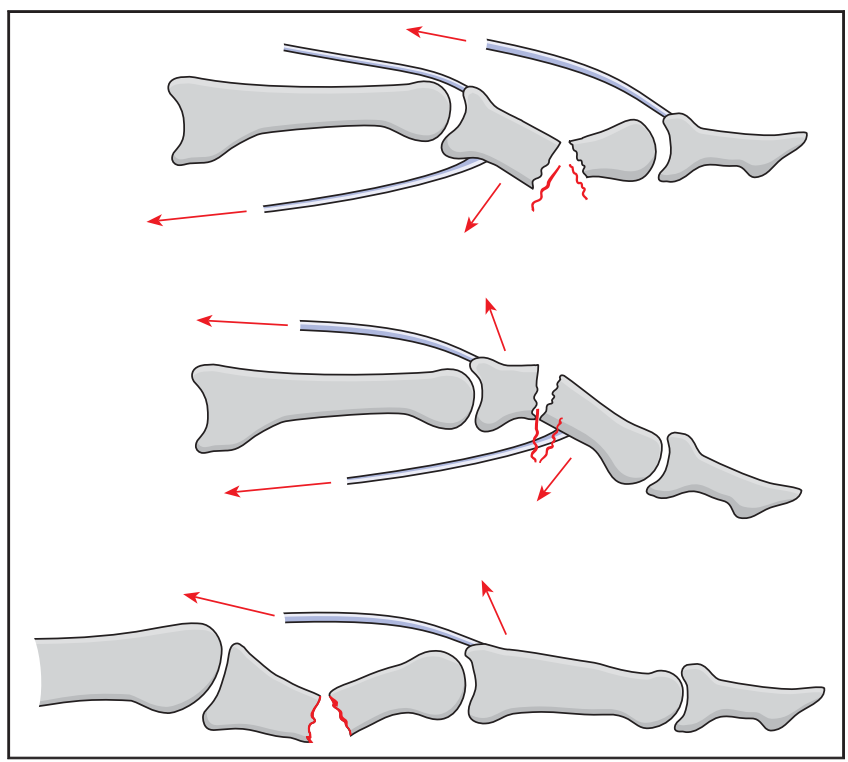

Abb. 10 Typische Fehlstellungen an den Phalangen bei einfachen Querfrakturen durch ansetzende Sehnen.

\section{Korrekturosteotomie an den Phalangen}

Operativer Zugang

Der Zugang richtet sich nach dem Verlauf der Osteotomie. Eine mediolaterale Inzision schont den Streckapparat an der Grundphalanx und ist gegenüber dem dorsalen Zugang zu bevorzugen. Dieser Zugang gestattet außerdem die Anlage von Minikondylenplatten von lateral. Die Exzision von Anteilen des Tractus lateralis erleichtert den Zugang und verhindert Adhäsionen über den Miniplatten sowie intrinsische Kontrakturen. Eine dorsale Plattenanlage über einen dorsalen Zugang kann jedoch wegen der besseren Anmodellierung der Platte günstig sein. In diesem Fall erfolgt nach dem Hautschnitt eine Spaltung (Splitting) des zentralen Anteils des Streckapparats im Verlauf des Tractus intermedius.
Der Zugang zur Mittelphalanx erfolgt über eine dorsale bogenförmige Inzision, die vom Mittelgelenk bis zum Endgelenk reicht. Der Ansatz des Tractus intermedius ist dringend zu schonen. Der Streckapparat lässt sich soweit mobilisieren, dass er zur Seite weggehalten werden kann. Bei der Osteotomie ist unbedingt auf eine Schonung der palmar liegenden Gefäßnervenbündel und der Beugesehnen zu achten.

\section{Osteotomieformen}

\section{Auf- und zuklappende Osteotomien}

Die Korrektur der fehlverheilten Phalanx erfolgt in analoger Weise wie am Mittelhandknochen (s.o.). Eine Achsabweichung in der Frontalebene beruht oft auf einem Knochendefekt des betroffenen Schaftes. Die operative Behandlung besteht in einer aufklappenden, inkompletten Osteotomie auf der Konkavseite des Knicks (Abb. 11). Die Osteotomie sollte die gegenüberliegende Kortikalis und 

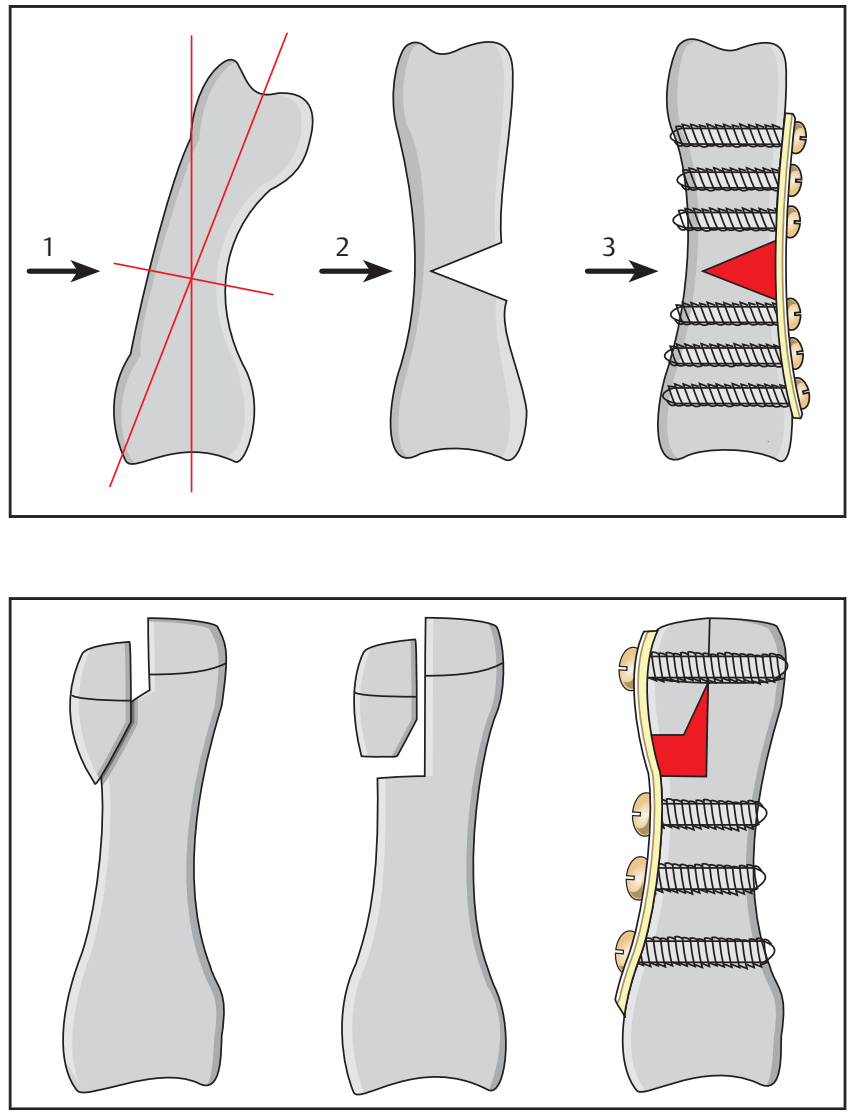
teotomie. Stufenfreie und Abstützplatte er-

das Periost stehen lassen, da dieser Bereich als Scharnier bei der Eröffnung der Osteotomie dienen kann, um die korrekte Angulation wiederherzustellen. Anschließend wird ein Knochentransplantat in den Defekt eingebracht und eine seitliche Abstützplatte angebracht. Drehfehler und Achsfehler können gleichzeitig korrigiert werden.

\section{Extraanatomische Korrektur der Rotationsfehlstellung}

Die Freilegung der ehemaligen Frakturstelle zur Korrekturosteotomie kann nach vorausgegangenen schweren Weichteilverletzungen ein erhebliches Risiko für die Weichteile bedeuten und durch neue narbige Adhäsionen die mühsam gewonnene Funktion wieder zunichtemachen. Bei einer isolierten Rotationsfehlstellung kann es vorteilhaft sein, die Korrektur nicht im ehemaligen Frakturbereich, sondern als derotierende Osteotomie proximal am zugehörigen Mittelhandknochen durchzuführen. Begleitende Achsabweichungen werden zwar nicht ausgeglichen, das Überkreuzen der Finger beim Faustschluss lässt sich jedoch zuverlässig durch diese Methode beheben.
Abb. 12 Intraartikuläre KorrekturosWiederherstellung der Gelenkfläche. Knochentransplantat

forderlich.

Abb. 11 Aufklaplanx. 1 = Verlauf der Osteotomie. 2 = stehengelassene Kortikalis auf der Konvexseite. 3 = Auffüllung mit Spongiosa. pende Korrekturos- eignet, die eine hochpräzise Fixation erlauben und stabil genug sind, um das Korrekturergebnis zu halten. Darüber hinaus müssen die eingesetzten Osteosyntheseverfahren eine Frühmobilisation erlauben und Nachbarstrukturen möglichst wenig irritieren. Nur so können Funktionseinbußen durch das erneute Trauma und eine längere Ruhigstellung vermieden werden. Auch dies setzt eine ausreichende Stabilität voraus. Einfache Osteosynthesen mit Kirschner-Drähten dürfen daher nur speziellen Indikationen, wie bspw. bei Kindern oder extrem kleinen Fragmenten, vorbehalten sein. In der Regel wird eine entsprechende Stabilität und Präzision nur durch Schrauben und Platten erreicht. Die modernen Miniplatten zeichnen sich durch ein niedriges Profil und eine geringe Kontaktfläche zum Knochen aus. Neuartige winkelstabile Platten lassen sich sowohl lateral als auch streckseitig anbringen. Frühzeitig erfasste artikuläre oder lange schräge Schaftfrakturen lassen sich im Frakturverlauf korrigieren und stabilisieren.

Ist eine Korrektur in einer Ebene ausreichend, so können bei zu- oder aufklappenden Osteotomien am Scheitelpunkt die Kortikalis und das Periost intakt bleiben. Dieser Bereich wirkt dann als Scharnier, das die Osteotomie stabilisiert und die versehentliche Erzeugung eines neuen Rotationsfehlers verhindert.

Bei Rotationsfehlstellungen ist eine vollständige Osteotomie erforderlich. Hier sind die technischen Anforderungen an die Präzision der Korrektur und auch der Fixation besonders hoch. Eine exzentrische Bohrung, die im Plattenloch um $1 \mathrm{~mm}$ versetzt ist, kann bereits eine Fehlrotation von $10^{\circ}$ verursachen. In diesem Bereich sollten die Schrauben daher nicht exzentrisch, sondern genau zentriert in den Plattenlöchern platziert werden.

Trotz aller technischen Fortschritte neigen die Miniplatten zur Verklebung mit darüberliegenden Gleitstrukturen und führen dann zu Bewegungseinschränkungen. In diesen Fällen sollte nach Ausheilung, frühestens nach Ablauf von 6 Monaten, die Implantatentfernung in Kombination mit Sehnen- und Gelenklösungen vorgenommen werden. Andererseits gewährleisten Miniplatten unmittelbar nach der Operation eine höhere Stabilität. Dadurch ist unmittelbar postoperativ eine intensive Übungsbehandlung möglich, die das Risiko von Verklebungen und Einsteifungen reduziert. 
Typische Komplikationen von Korrekturosteotomien an der Hand sind

- persistierende Fehlstellung

- verzögerte Knochenheilung

- Implantatversagen (Plattenbruch, Schraubenlockerung, Implantatwanderung)

- Infektionen

- anhaltende oder verstärkte Bewegungseinschränkungen und Steifigkeit - dystrophe Reaktionen

- persistierende Schmerzen

\section{Schlussfolgerungen}

Verbliebene posttraumatische Fehlstellungen an der Hand können zu erheblichen Funktionsbeeinträchtigungen führen. Neben den Grundformen der Verkürzung, der Achsabweichung und des Rotationsfehlers kommen häufig kombinierte Formen vor. Korrekturosteotomien können diese Fehlstellungen beseitigen. Grundsätzlich hängt die Durchführung von den Bedürfnissen des Patienten ab. Dabei sind Alter, Aktivitätsniveau und Anspruch des Betroffenen zu berücksichtigen.
Sie sollte möglichst frühzeitig, d.h. innerhalb der ersten 10 Wochen nach dem Unfallereignis, durchgeführt werden, weil zu diesem Zeitpunkt noch eine Korrektur im Frakturverlauf möglich ist, das Kallusgewebe noch relativ weich ist und funktionsbehindernde Kontrakturen und Fibrosen sich noch nicht entwickelt haben.

Zuklappende Osteotomien haben den Vorteil, dass in der Regel kein Knochentransplantat erforderlich ist und nur eine Osteotomie knöchern ausheilen muss. Eine Verkürzung kann dadurch allerdings nicht ausgeglichen werden. Sollte dies erforderlich sein, so bietet sich eine aufklappende Osteotomie an, die allerdings eine Defektauffüllung mit Knochentransplantaten und eine stabile osteosynthetische Versorgung erfordert. Die Beseitigung von Rotationsfehlern ist besonders anspruchsvoll, weil hier bereits geringe Abweichungen das Korrekturergebnis zunichte machen können. Durch intraartikuläre Korrekturosteotomien ist es möglich, die Kongruenz der Gelenkflächen wiederherzustellen.

\section{Literatur}

1 Balaram AK, Bednar MS. Complications after the fractures of metacarpal and phalanges. Hand Clin 2010; 26: 169-177

2 Dautel G. Fehlstellungen und Pseudarthrosen an den Metakarpophalangealknochen. In: Merle M, Dautel G, Rehart S, Hrsg. Chirurgie der Hand. Stuttgart: Thieme; 1999: 3-10

${ }^{3}$ Day CS, Stern PJ. Fractures of the Metacarpals and Phalanges. In: Wolfe S, Hotchkiss R, Pederson W et al., eds. Green's operative Hand Surgery. 6th ed. Philadelphia: Churchill Livingstone; 2011: 239-290

${ }^{4}$ Freeland AE, Lindley SG. Malunions of the finger metacarpals and phalanges. Hand Clin 2006; 22: 341-355

${ }^{5}$ Pechlaner S, Hussl H, Kerschbaumer F. Behandlung fehlverheilter Verletzungen. In: Pechlaner S, Hussl H, Kerschbaumer F, Hrsg. Operationsatlas Handchirurgie. Stuttgart, New York: Thieme; 1998: 322-325

${ }^{6}$ Rudigier J, Hrsg. Kurzgefasste Handchirurgie. 5. Aufl. Stuttgart: Thieme; 2006

7 Seitz jr. WH, Froimson AI. Management of malunited fractures of the metacarpal and phalangeal shafts. Hand Clin 1988; 4: 529536

${ }^{8}$ Teoh LC, Yong FC, Chong KC. Condylar advancement osteotomy for correcting condylar malunion of the finger. J Hand Surg $[\mathrm{Br}]$ 2002; 27: 31-35

9 Weckesser EC. Rotational osteotomy of the metacarpal for overlapping fingers. J Bone Joint Surg [Am] 1965; 47: 751-756

\section{Dr. med. Thomas Pillukat \\ Facharzt für Chirurgie \\ Dr. med. Karlheinz Kalb \\ Facharzt für Chirurgie \\ Prof. Dr. Jörg van Schoonhoven \\ Chefarzt}

Klinik für Handchirurgie

Salzburger Leite 1

97616 Bad Neustadt an der Saale

t.pillukat@handchirurgie.de 